\title{
Direct measurement of the Hall factor for holes in relaxed $\mathrm{Si}_{1-x} \mathbf{G e}_{\boldsymbol{x}}(0<X<1)$
}

\author{
Y. C. Chen, S. H. Li, P. K. Bhattacharya, J. Singh, and J. M. Hinckley \\ Department of Electrical Engineering and Computer Science, Solid-State Electronics Laboratory, \\ University of Michigan, Ann Arbor, Michigan 48109-2122
}

(Received 4 January 1994; accepted for publicalion 14 March 1994)

The Hall factor for holes in relaxed $p$-type $\mathrm{Si}_{1-x} \mathrm{Ge}_{x}$ alloys has been determined from mobility measurements at magnetic fields up to $7 \mathrm{~T}$ at $290 \mathrm{~K}$. Our data together with previously published values for $\mathrm{Si}$ and $\mathrm{Ge}$ suggest that $r$ for holes in SiGe varies between 0.73 and 1.7 with a possible strong bowing.

Coherently strained $\mathrm{Si}_{1-x} \mathrm{Ge}_{x}$ alloys and $\mathrm{SiGe} / \mathrm{Si}$ heterostructures are currently of immense interest due to their potentially useful electronic and optical properties and compatibility with existing $\mathrm{Si}$ technology. ${ }^{1-3}$ The built-in mismatch strain dramatically alters the band-structure and carrier transport properties. Although some reports of improved device performance in this material system have been made, numerous important experimental issues in transport of both minority and majority carriers are still not clear.

For the calculation of low- and high-field transport properties of semiconductors, and for the analysis of transport data, knowledge of the Hall factor $r$ is essential. The value of $r$, which is the ratio of the Hall mobility $\mu_{H}$ to the drift mobility $\mu_{d}$, is generally unity if carriers are confined in a single minimum. However, in multivalleyed systems, the value of $r$ can deviate significantly from unity. For example, for a two-band (heavy and light holes) valence band system, the Hall factor $r$ bears the following relation to the individual band Hall factors $r_{\mathrm{hh}}$ and $r_{\mathrm{lh}}:^{4}$

$$
r=\frac{f_{\mathrm{hh}} r_{\mathrm{hh}} \mu_{\mathrm{hh}}^{2}+f_{\mathrm{lh}} r_{\mathrm{lh}} \mu_{\mathrm{hh}}^{2}}{\left(f_{\mathrm{hh}} \mu_{\mathrm{hh}}+f_{\mathrm{lh}} \mu_{\mathrm{lh}}\right)^{2}},
$$

where $f_{\mathrm{hh}}$ and $f_{\mathrm{lh}}$ are the fractions of carriers in the heavy hole and light hole bands, and $\mu_{\mathrm{hh}}$ and $\mu_{\mathrm{lh}}$ are the corresponding heavy and light hole mobilities. The individual quantities are calculated accordingly:

$$
r_{\mathrm{hh}}=\frac{\left\langle\tau_{\mathrm{hh}}^{2}\right\rangle}{\left\langle\tau_{\mathrm{hh}}\right\rangle^{2}}, \quad \mu_{\mathrm{hh}}=\frac{e\left\langle\tau_{\mathrm{hh}}\right\rangle}{m_{\mathrm{hh}}^{*}}, \quad f_{\mathrm{hh}}=\frac{n_{\mathrm{hh}}}{n_{\mathrm{hh}}+n_{\mathrm{lh}}},
$$

with corresponding expressions for the light hole terms. The average of the $n$th power of the relaxation time $\tau$ is ${ }^{5}$

$$
\left\langle\tau_{\mathrm{hh}}^{n}\right\rangle=\frac{\int_{0}^{\infty} \tau^{n}(E) D_{\mathrm{hh}}(E) E \exp (-E / k T) d E}{\int_{0}^{\infty} D_{\mathrm{hh}}(E) E \exp (-E / k T) d E},
$$

where $D_{\mathrm{hh}}(E)$ is the heavy hole density of states. A similar expression pertains to the light hole relaxation time.

The value of $r \sim 1$ in the conduction band of $\mathrm{Si}, \mathrm{Ge}$, and the resulting mixed alloys. ${ }^{6}$ Values of $r=0.73$ in $\mathrm{Si}^{7}$ and $r=1.7$ in $\mathrm{Ge}^{8}$ have been reported for holes. To our knowledge, there is no report of any direct measurement of $r$ for holes in $\mathrm{Si}_{1-x} \mathrm{Ge}_{x}$. In this letter, we report the measurement of $r$ in the valence bands of SiGe alloys grown by gas-source molecular beam epitaxy.
The experimental $\mathrm{Si}_{1-x} \mathrm{Ge}_{x}$ samples were grown on high resistivity (>1000 $\Omega \mathrm{cm}$ ) (001)Si substrates by molecular beam epitaxy using gaseous disilane $\left(\mathrm{Si}_{2} \mathrm{H}_{6}\right)$ and solid $\mathrm{Ge}$ as sources. Details of our growth system and procedures have been published elsewhere. ${ }^{9}$ The samples were doped $p$ type with solid $B$ at a level of $10^{17} \mathrm{~cm}^{-3}$ and were each approximately $1 \mu \mathrm{m}$ thick. The composition of the alloy films were confirmed by double-crystal $\mathrm{x}$-ray measurements. Crosssectional transmission electron microscopy measurements on these samples show that most of the misfit dislocations are contained and localized at the substrate-epilayer interface. We therefore believe that the transport measurements reflect the intrinsic properties of SiGe.

Measurements were made at $290 \mathrm{~K}$ in an Oxford superconducting magnet in which a maximum magnetic field of 7 $\mathrm{T}$ is obtained. The experimental van der Pauw samples were defined by standard photolithography. The ohmic contacts on these samples were formed by evaporation of $300 \mathrm{~nm} \mathrm{Al}$ and subsequent annealing at $400^{\circ} \mathrm{C}$ for $3 \mathrm{~min}$. Typical contact resistances are approximately $\sim 10^{-6} \Omega \mathrm{cm}^{2}$.

It can be shown ${ }^{5}$ that if the magnetic flux density $B$ is large enough, then the measured mobility in the van der Pauw determination is in fact the true drift mobility. This condition is easily satisfied for electrons at moderate fields and is usually satisfied for holes for $B \sim 10 \mathrm{~T}$. Measured data in two samples are shown in Fig. 1. It is assumed that the saturation behavior at the highest magnetic field corresponds to $\mu_{H}=\mu_{d}$. It is evident that at $B=0$ or a small value, $r<1$ for $x=0.22$ and $r>1$ for $x=0.35$. The measured values of $r$

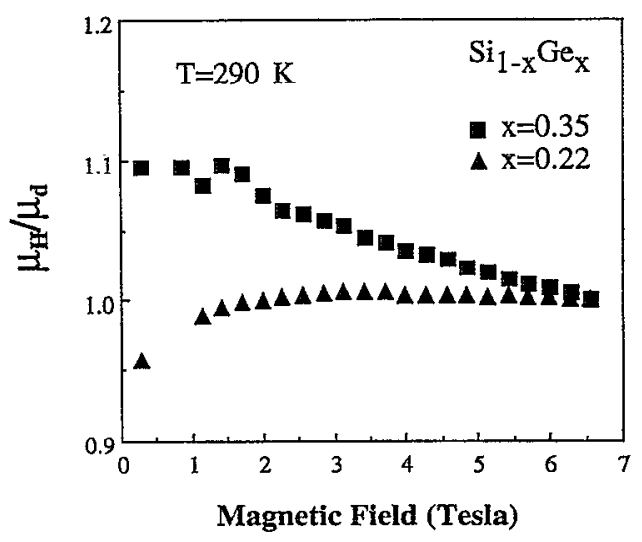

FIG. 1. Measured Hall Scattering factor vs magnetic field in $\mathrm{Si}_{1-x} \mathrm{Ge}_{x}$. 


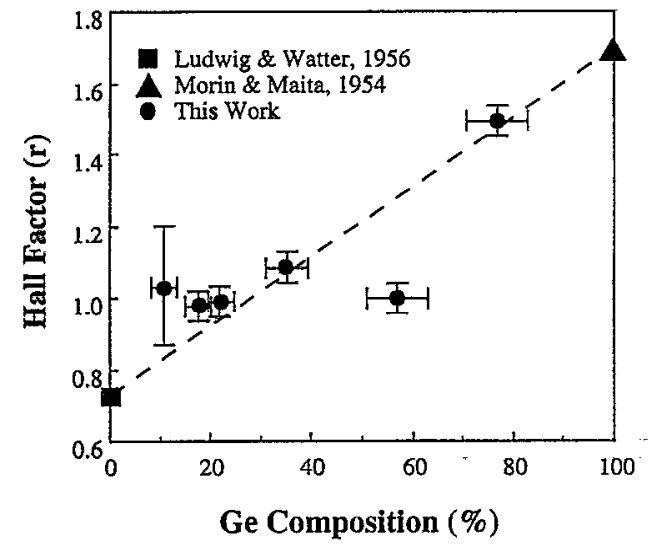

FIG. 2. Variation of measured Hall factor with alloy composition in $\mathrm{Si}_{1-x} \mathrm{Ge}_{x}$ (note that the vertical error bar for $x=0.11$ is larger than the rest of the data due to anomalous fluctuations in the field during measurements with this sample).

in all the samples are plotted in Fig. 2 together with the reported data for $\mathrm{Si}(x=0)$ and $\mathrm{Ge}(x=1)$. The dashed line indicates a linear interpolation between the $\mathrm{Si}$ and $\mathrm{Ge}$ data. It is clear that the experimental data indicates $r \sim 1$ up to $x=0.6$, beyond which $r>1$. The apparent bowing in the data may partly be due to large alloy scattering for holes in these materials, as mentioned later.

The variation of the Hall factor in the alloys was also estimated theoretically, using Eqs. (1)-(3). The relaxation time was approximated as the inverse of the total scattering rate. The theoretical results show a linear variation of the Hall factor with composition although the absolute magnitude of the Hall factor was found to be about a factor of 2 too large overall. This is to be expected as a consequence of the approximation of using spherical bands with anisotropic scattering. ${ }^{8}$ We therefore believe that the Hall factor for holes varies in some complex manner between the values of $\mathrm{Si}$ and Ge. The measured low-field hole mobilities in the same samples using the Hall factors are shown in Fig. 3. The strong bowing is due to the very strong alloy scattering in these materials. In fact, a value of the alloy scattering potential $U_{0}=0.6 \mathrm{eV}$ has been measured from analysis of high ${ }^{10}$ and low ${ }^{11}$ field transport data.

The Hall scattering factor for holes in very heavily doped (approximately $10^{19} \mathrm{~cm}^{-3}$ ) $\mathrm{Si}_{1-x} \mathrm{Ge}_{x}$ was reported recently by McGregor et al. ${ }^{12}$ Their data indicate a decreasing value of $r$ with increasing $x$, in clear contrast to our data. This trend would also not support the earlier published values of $r$ in Si and Ge. However, these authors extract the value of $\mu_{d}$ from sheet resistance measurements of the heavily doped base regions of $n-p-n$ bipolar transistors. In these samples the system is nearly degenerate and therefore the $r$ factor approaches unity as in metals.

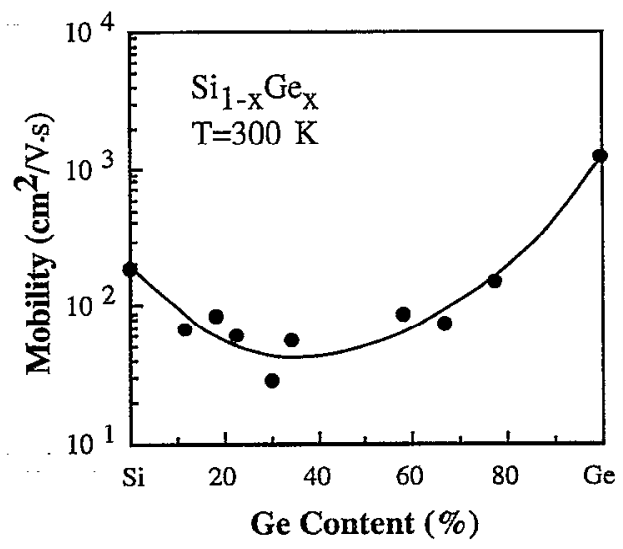

FIG. 3. Variation of measured Hall mobility with alloy composition in p-type $\mathrm{Si}_{1-x} \mathrm{Ge}_{x}$.

The data reported in this letter are for relaxed materials and thereby represent the intrinsic properties of the alloys. In a pseudomorphically strained $\mathrm{Si}_{1-x} \mathrm{Ge}_{x}$ alloy, the strain lifts the hh and $\mathrm{lh}$ degeneracy and therefore much of the carriers occupy the hh band only. Also, the hh band becomes quite light and is more parabolic. Thus, the behavior of the Hall factor may be quite different for the strained $\mathrm{Si}_{1-x} \mathrm{Ge}_{x}$ system.

In conclusion, we report the first direct measurement of the Hall factor for holes in $\mathrm{Si}_{1-x} \mathrm{Ge}_{x}$. The measured values vary between the values for $\mathrm{Si}(0.73)$ and $\mathrm{Ge}(1.7)$ with a strong bowing.

The work is supported by the U.S. Air Force Office of Scientific Research and the Materials Research Laboratory, Wright-Patterson Air Force Base, under Grant No. AFOSR91-0349.

${ }^{1}$ G. L. Patton, J. H. Comfort, B. S. Meyerson, E. F. Crabbe, G. J. Scilla, E. D. Fresart, J. M. C. Stork, J. Y. C. Sun, D. L. Harame, and J. N. Burghartz, IEEE Trans. Electron Device Lett. 11, 171 (1990).

${ }^{2}$ K. Ismail, B. S. Meyerson, S. Rishton, J. Chu, S. Nelson, and J. Nocera, IEEE Trans. Electron Device Lett. 13, 229 (1992).

${ }^{3}$ Q. Mi, X. Xiao, J. C. Sturm, L. C. Lenchyshyn, and M. L. W. Thewalt, Appl. Phys. Lett. 60, 3177 (1992).

${ }^{4}$ H. Fritzsche, Methods of Experimental Physics (Academic, New York, 1959), Vol. 6, Part B, Chap. 8.

${ }^{5}$ D. C. Look, Semiconductors and Semimetals (Academic, New York, 1983), Vol. 19, Chap. 2.

${ }^{6} \mathrm{~J}$. Singh, Physics of Semiconductors and Their Heterostructures (McGraw-Hill, New York, 1993), p. 340.

${ }^{7}$ G. W. Ludwig and R. L. Watters, Phys. Rev. B 101, 1699 (1956).

${ }^{8}$ F. Morin and J. Maita, Phys. Rev. 96, 28 (1954).

${ }^{9}$ S. H. Li, P. K. Bhattacharya, R. Malik, and E. Gulari, J. Electron. Mater. 22, 793 (1993).

${ }^{10}$ S. H. Li, J. M. Hinckley, J. Singh, and P. K. Bhattacharya, Appl. Phys. Lett. 63, 1393 (1993).

${ }^{11}$ V. Venkataraman, C. W. Liu, and J. C. Sturm, Appl. Phys. Lett. 63, 2795 (1993).

${ }^{12}$ J. M. McGregor, T. Manku, J. P. Noel, D. J. Roulston, A. Nathan, and D. C. Houghton, J. Electron. Mater. 22, 319 (1993). 